\title{
Resonant-state expansion applied to planar waveguides
}

\author{
L. J. Armitage, M. B. Doost, W. Langbein, and E. A. Muljarov* \\ School of Physics and Astronomy, Cardiff University, Cardiff CF24 3AA, United Kingdom
}

(Received 21 October 2013; published 27 May 2014)

\begin{abstract}
The resonant-state expansion, a recently developed method in electrodynamics, is generalized here to planar open optical systems with non-normal incidence of light. The method is illustrated and verified on exactly solvable examples, such as a dielectric slab and a Bragg reflector microcavity, for which explicit analytic formulas are obtained. This comparison demonstrates the accuracy and convergence of the method. Interestingly, the spectral analysis of a dielectric slab, in terms of resonant states, reveals an influence of waveguide modes in the transmission. These modes, which on-resonance do not couple to external light, surprisingly do couple to external light for off-resonant excitation.
\end{abstract}

DOI: 10.1103/PhysRevA.89.053832

PACS number(s): 42.25.-p, 03.50.De, 03.65.Nk

\section{INTRODUCTION}

Optical waveguides (WGs) are a basic building block for optical technology owing to their lossless guiding of light, enabled, for example, by total internal reflection. WGs provide confinement of the light in one or two dimensions, whereas, allowing light waves to propagate along the remaining dimensions in which the waveguides are approximately invariant. Planar WGs with one-dimensional (1D) confinement, such as a dielectric slab, and fiber WGs with two-dimensional (2D) confinement, are widely used, for example, in fiberoptic cables for telecommunication, photonic crystal fibers [1], integrated optical circuits [2], and terabit chip-to-chip interconnects [3].

The optical spectra of WGs, however, do not consist of only these bound modes called WG modes, but also contain unbound modes which couple to the outside, commonly known as leaky modes. An elegant and intuitive way to understand and describe the properties of optical systems is to use the concept of discrete resonant states (RSs) [4,5], which include all types of modes in the system and present a mathematically complete set of spatial functions. RSs are defined as eigensolutions of the Maxwell equations having outgoing wave boundary conditions (BCs). Their energies are generally complex with the real and imaginary parts, respectively, corresponding to the spectral position and the linewidth of the mode. The quality factor of the mode is given by the real part divided by twice the imaginary part of the mode frequency. The finite imaginary part reflects the fact that these states decay in time and leak out of the system. Such RSs grow exponentially outside of the system and require an adapted normalization [4-9]. WG modes, which may exist in the system, are included in the set of RSs and are required for the completeness of the set, even though they have real energies and evanescent tails.

To calculate the RSs in optical systems in which analytic solutions are not possible, the resonant-state expansion (RSE), a rigorous perturbation method in electrodynamics, has recently been developed [6] and has been applied to finite 1D, two-dimensional (2D), and three-dimensional (3D) systems, such as planar [7], cylindrical [8], and spherical [9] resonators with perturbations, respectively. The RSE turned

\footnotetext{
*egor.muljarov@astro.cf.ac.uk
}

out to be suited for the calculation of sharp resonances, such as whispering gallery modes. Such modes have previously been investigated in microcylinders [10-12] and microspheres [13]. Available computational techniques, such as the finite difference in time domain (FDTD) $[14,15]$ and finite element method (FEM) [16-18] need large computational resources $[19,20]$ to calculate such modes. Very recently, we have compared the performance of the RSE with commercial FEM and FDTD solvers and have found that the RSE was a few orders of magnitude more computationally efficient [9]. It, therefore, has the potential to supersede the presently used methods.

Up to now, the RSE has been applied only to modes with zero wave vector $p$ along the translationally invariant direction of the system considered, corresponding to normal incidence of light without propagation along the waveguide. In this paper, we extend the application of RSE to arbitrary wave vectors $p$, thus, allowing to describe the propagation along waveguide structures. This introduces in the spectrum of RSs, which, for normal incidence, is dominated by lossy Fabry-Pérot (FP) modes, WG and antiwaveguide (AWG) modes, as well as a continuum of modes due to a cut of the Green's function (GF) in the complex frequency plane appearing for $p \neq 0$. The modes on the cut contribute significantly to the optical spectra and are required for the completeness of the RS basis. They present a challenge in the technical implementation of the $\mathrm{RSE}$, which is dealing with discrete states. We have recently shown [8] that one can perform an effective discretization of such continua for the RSE applied to 2D systems which already show a cut for $p=0$.

In the present paper, we eliminate the cut in planar systems with $p \neq 0$ by going from the frequency representation of the system to the normal wave-vector representation. We treat planar WGs and verify our theory on exactly solvable structures, such as a homogeneous dielectric slab and a Braggmirror microcavity (MC), using the RSs of a homogeneous slab as a basis for the RSE. The role of the different types of RSs is studied in detail, revealing the importance of WG modes in the transmission. The method of eliminating the cut presented here can be applied to fiber WGs, generalizing our recent paper on cylindrical resonators [8] to non-normal incidence and will be the subject of a future paper. Furthermore, it enables treating planar photonic crystals and cavities, which have important applications. Given the superior performance of RSE in 3D 
systems [9], we expect that the RSE has the potential to improve on presently available approaches which are based on the WG mode expansion [21,22] or on scattering matrix formalism [23,24].

The paper is organized as follows. In Sec. II, we study the transmission of a homogeneous slab in the complex frequency and normal wave-vector plane in order to analyze the contributions of different types of RSs to the optical spectra of planar WGs. In Sec. III, we present a general formulation of the RSE for planar systems with nonzero in-plane momentum. In Sec. IV, we demonstrate applications of the RSE to different systems and compare results with available exact solutions. In particular, in Sec. IV A, we introduce the basis of RSs for a homogeneous slab in inclined geometry and then use it for calculation of optical modes of a homogeneous slab with a different refractive index in Sec. IV B, and of a Bragg-mirror microcavity in Sec. IV C.

\section{ROLE OF WAVEGUIDE MODES IN TRANSMISSION SPECTRA}

We study the role of RSs in the transmission of a dielectric slab and, in particular, the influence of the WG modes on the slab transmission. The WG modes are RSs which have zero linewidth and are present in the spectrum of a planar system at non-normal incidence of the incoming light wave. We consider a dielectric slab with thickness $2 a$ in vacuum, having the real dielectric constant,

$$
\varepsilon(z)=\left\{\begin{array}{lll}
\epsilon_{s} & \text { for } & |z| \leqslant a, \\
1 & \text { for } & |z|>a,
\end{array}\right.
$$

where $\epsilon_{s}$ is the permittivity of the slab and $z$ is the coordinate normal to the slab. We assume a permeability of $\mu=1$ everywhere throughout this paper. The electric field $\mathbf{E}(\mathbf{r}, t)$ satisfies Maxwell's wave equation,

$$
\nabla \times \nabla \times \mathbf{E}+\varepsilon(z) \frac{1}{c^{2}} \frac{\partial^{2} \mathbf{E}}{\partial t^{2}}=0
$$

and Maxwell's BCs on the dielectric-vacuum interfaces. For an incoming plane monochromatic wave with the transverseelectric (TE) polarization along $\hat{y}$ ( $\hat{y}$ is the unit vector along the $y$ axis) and real frequency $\omega$, the electric field in the system takes the form

$$
\mathbf{E}(\mathbf{r}, t)=\hat{y} e^{-i \omega t+i p x} E(z),
$$

in which $p$ is the in-plane projection of the wave vector. For the component $E(z)$ of the electric field, Eq. (2) transforms to a $1 \mathrm{D}$ wave equation,

$$
\left[\frac{d^{2}}{d z^{2}}-p^{2}+\varepsilon(z) \frac{\omega^{2}}{c^{2}}\right] E(z)=0 .
$$

The electric field for $z>a$ is given by the transmitted plane wave $E(z)=t(\omega) e^{i k z} E_{0}$, where $E_{0}$ is the amplitude of the incoming wave. The field transmission through the slab $t(\omega)$ has the analytic form

$$
t(\omega)=\frac{2 i k q e^{-2 i k a}}{2 i k q \cos (2 q a)+\left(k^{2}+q^{2}\right) \sin (2 q a)}=T(k),
$$

in which

$$
\begin{gathered}
k=\sqrt{\left(\frac{\omega}{c}\right)^{2}-p^{2}} \\
q=\sqrt{\epsilon_{s}\left(\frac{\omega}{c}\right)^{2}-p^{2}}=\sqrt{\epsilon_{s} k^{2}+\left(\epsilon_{s}-1\right) p^{2}}
\end{gathered}
$$

are the $z$ components of the wave vector in vacuum and dielectric, respectively. Equation (5) shows that the transmission $t(\omega)$ is a function of the real frequency $\omega$. One can also express the transmission $t(\omega)$ as a function $T(k)$ of the normal wave vector $k$ in which $k$ takes only real positive values as dictated by the outgoing character of the transmitted wave. The wave vector $q$ inside the slab can be complex for a dielectric with dissipation and can have an arbitrary sign, reflecting the fact that waves within the slab propagate in both directions. Hence, the transmission is insensitive to the sign of $q$ as seen in Eq. (5).

To study the influence of different modes on the transmission, we consider analytic continuations (ACs) $\tilde{t}(\omega)$ and $\tilde{T}(k)$ of both functions in the complex $\omega$ and $k$ planes, respectively, in order to investigate their pole structures and for each of them, apply the Mittag-Leffler theorem [25-27]. The AC of the transmission has different types of poles, which are shown in Fig. 1 for $p a=5$. As in the case of normal incidence [7], there is a countable-infinite number of FP modes having nearly equidistant real parts and finite imaginary parts. In addition, there are two types of modes on the real $\omega$ axis: WG and AWG modes, which are appearing for $p \neq 0$. The WG modes have an evanescent, i.e., exponentially decaying electric field into the vacuum, whereas, the AWG modes are exponentially growing into the vacuum and are known in quantum mechanics as antibound states [28]. Finally, there is one leaky mode (LM) at the center of the spectrum which has zero real and negative imaginary parts of $\omega$. Due to the square root in Eq. (6), the function $\tilde{t}(\omega)$ has two branch points at $\omega= \pm p c$ connected by a cut. We choose the cut going through $\omega=-i \infty$ and,

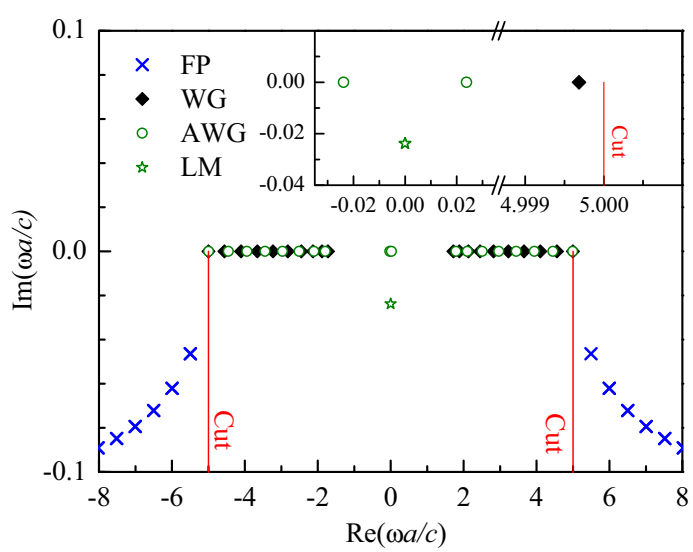

FIG. 1. (Color online) Poles (symbols) and cut (red lines) of the transmission $\tilde{t}(\omega)$ of a homogeneous dielectric slab with $\epsilon_{s}=9$ and in-plane wave vector $p a=5$. The poles are Fabry-Pérot (blue crosses), waveguide (black diamonds), and antiwaveguide modes (open circles), including a leaky mode (open star). The inset shows the absence of $\omega=0$ and $k=0$ modes. 
thus, producing two vertical cut lines as shown in Fig. 1. The other square root in the definition of $q(\omega)$ does not produce any cuts due to the above-mentioned fact that $t(\omega)$ is an even function of $q$ and, thus, independent of its sign. Integrating $\tilde{t}\left(\omega^{\prime}\right) /\left(\omega-\omega^{\prime}\right)$ over a closed infinite-radius circular contour circumventing the cut, similar to that used in Ref. [8], we obtain the spectral representation in the frequency domain,

$$
\tilde{t}(\omega)=\sum_{n} \frac{\operatorname{Res}_{\omega^{\prime}=\omega_{n}}\left[\tilde{t}\left(\omega^{\prime}\right)\right]}{\omega-\omega_{n}}+\frac{1}{2 \pi i} \sum_{p^{\prime}= \pm p} \int_{p^{\prime} c-i \infty}^{p^{\prime} c} \frac{\Delta t\left(\omega^{\prime}\right) d \omega^{\prime}}{\omega-\omega^{\prime}} .
$$

Here the first term represents a sum over residues at all poles of $\tilde{t}(\omega)$. The second term is the integral of the step $\Delta t(\omega)$ in the transmission along the two parts of the cut shown in Fig. 1. Specifically, $\Delta t(\omega)$ is defined as the difference between the values of $\tilde{t}(\omega)$ on the left and right sides of the cut for the given cut point $\omega$.

Using the spectral representation Eq. (8) for real frequencies $\omega$, we analyze contributions of the poles and the cut to the transmission. The transmission is usually studied for a fixed angle of incidence $\theta$, motivated by experimental constraints. An example of the calculated transmission through a slab with $\epsilon_{s}=9$ is shown for $\theta=\pi / 4$ in Fig. 2(a). For a fixed $\theta$, the
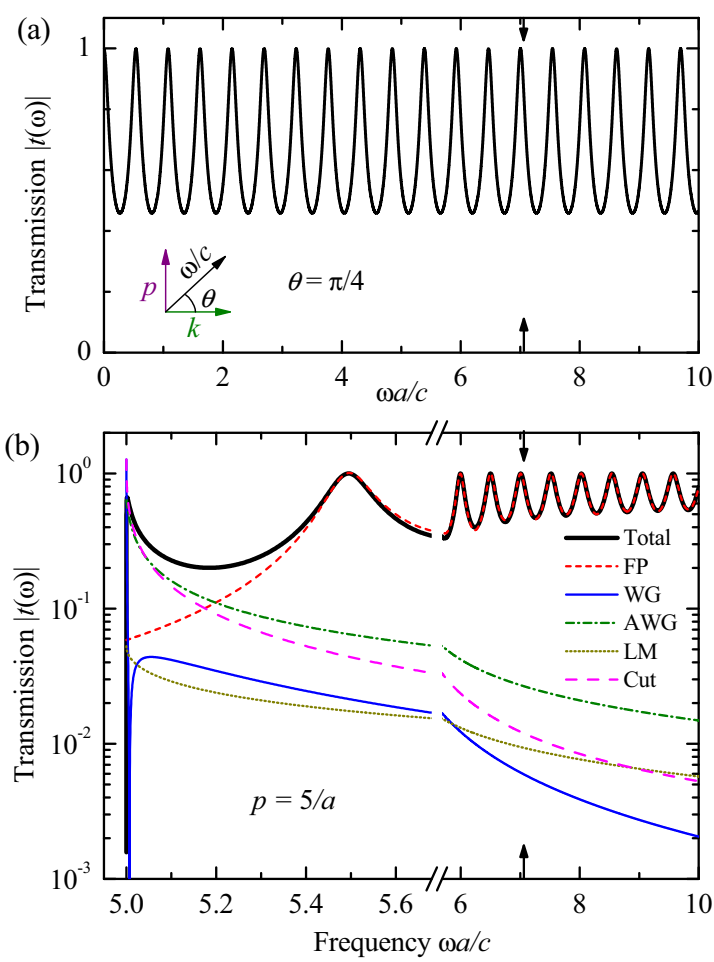

FIG. 2. (Color online) Transmission $|t(\omega)|$ of a homogeneous dielectric slab with $\epsilon_{s}=9$ as a function of the light frequency $\omega$, in (a) for a fixed angle of incidence $\theta=\pi / 4$ and in (b) for a fixed in-plane wave vector $p a=5$, along with partial contributions to the transmission of different types of modes and the cut shown in Fig. 1. Black vertical arrows indicate the frequency for which $p a=5$ in panel (a) and $\theta=\pi / 4$ in panel (b). The inset shows a schematic of the total wave vector $\omega / c$ along with its projections $p$ and $k$ on the $x$ and $z$ axes, respectively. in-plane wave vector $p$ changes with frequency so that the contributions of the poles (which are different for different $p$ 's) are not constant across the spectrum. We, therefore, analyze the spectrum for a fixed $p$ as shown in Fig. 2(b) in which the contributions of different pole types and the cut are shown individually, summing up to the analytic transmission Eq. (5). Note that the transmission $t(\omega)$ is defined over the angle range $0<\theta<\pi / 2$, corresponding to $\omega>p c$. FP modes dominate for $\omega \gg p c$, giving rise to the oscillations in the transmission, whereas, the contribution of all other modes and the cut are significant only close to the threshold $\omega=p c$, corresponding to grazing incidence $\theta \sim \pi / 2$.

The cut contribution to the spectral representation Eq. (8) and to the transmission in Fig. 2(b) produces a continuum of resonances. Such a continuum can approximately be treated in the RSE by replacing it with a series of poles as done in Ref. [8]. In the present case, however, the cut can actually be removed by going into the wave-vector domain. Indeed, being treated as a function of the normal wave vector $k$, the AC of the transmission $\tilde{T}(k)$ has no cuts in the complex $k$ plane, and its spectral representation, obtained by using the Mittag-Leffler theorem, has the following form:

$$
\tilde{T}(k)=\sum_{n} \frac{\operatorname{Res}_{k^{\prime}=k_{n}}\left[\tilde{T}\left(k^{\prime}\right)\right]}{k-k_{n}},
$$

in which $k_{n}=\sqrt{\omega_{n}^{2} / c^{2}-p^{2}}$ with $n$ numbering the poles as in Eq. (8). On the real $k$ axis, $\tilde{T}(k)$ coincides with the transmission $T(k)$ given by Eq. (5) and is shown in Fig. 3 along with the contributions of the different types of modes. We see, in particular, that the WG modes, which are not emitting into an outgoing plane wave, and thus, by reciprocity are expected not to be excitable by an incoming plane wave, have a finite contribution to the transmission, which is possible only due to their off-resonant excitation. This contribution increases with decreasing the wave vector $k$ as the frequency of the incoming wave is getting closer to the resonant frequencies of the WG modes lying beyond the vacuum light cone.

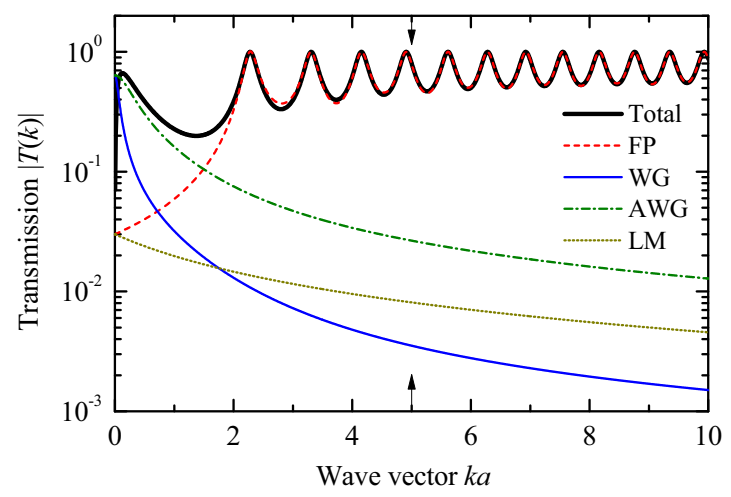

FIG. 3. (Color online) Transmission $|T(k)|$ of a homogeneous dielectric slab with $\epsilon_{s}=9$ and $p a=5$ and partial contributions of different modes as functions of the normal component $k$ of the wave vector in vacuum. As in Fig. 2(b), vertical arrows indicate the wave vector at which $\theta=\pi / 4$. 


\section{RESONANT-STATE EXPANSION FOR NON-NORMAL INCIDENCE}

The RSE formulated in our previous papers [6-8] is based on three key elements, which are as follows: (i) Dyson's equation for the GF, (ii) spectral representation of the GF, and (iii) completeness of RSs used for expansions of perturbed states and the GF itself. We have previously applied the RSE to infinite $1 \mathrm{D}$ and $2 \mathrm{D}$ systems at normal incidence. The nonnormal incidence, characterized by $p \neq 0$, is treated here. The previously used spectral representation of the GF in the frequency domain contains a cut for $p \neq 0$, which, however, can be removed by mapping the problem onto the complex normal wave-vector space $k$ as demonstrated in Sec. II. We, therefore, reformulate the RSE in the complex $k$ plane in which the spectral representation of the GF of an infinite planar system with an in-plane momentum $p \neq 0$ for TE polarization [29] has the form

$$
G_{k}\left(z, z^{\prime}\right)=\sum_{n} \frac{E_{n}(z) E_{n}\left(z^{\prime}\right)}{2 k_{n}\left(k-k_{n}\right)},
$$

where $E_{n}(z)$ is the electric field of a RS, defined as an eigensolution of Eq. (4) with an arbitrary profile of $\varepsilon(z)$ within a finite interval $|z|<a$, satisfying the outgoing wave $\mathrm{BCs}$,

$$
E_{n}(z) \propto e^{i k_{n}|z|} \text { for }|z|>a,
$$

and orthonormality conditions [30],

$$
\begin{aligned}
& \int_{-a}^{a} \varepsilon(z) E_{n}(z) E_{m}(z) d z \\
& -\frac{E_{n}(-a) E_{m}(-a)+E_{n}(a) E_{m}(a)}{i\left(k_{n}+k_{m}\right)}=\delta_{n m} .
\end{aligned}
$$

Substituting Eq. (10), which is valid for $|z|,\left|z^{\prime}\right|<a$, into the equation for the GF,

$$
\left[\frac{d^{2}}{d z^{2}}-p^{2}+\varepsilon(z)\left(k^{2}+p^{2}\right)\right] G_{k}\left(z, z^{\prime}\right)=\delta\left(z-z^{\prime}\right),
$$

using the wave equation for the eigenmodes, and taking the limit $k \rightarrow \infty$, we obtain the closure relation, $\varepsilon(z) \sum_{n} E_{n}(z) E_{n}\left(z^{\prime}\right)=2 \delta\left(z-z^{\prime}\right)$, and the sum rule:

$$
\sum_{n} \frac{E_{n}(z) E_{n}\left(z^{\prime}\right)}{k_{n}}=0 .
$$

For $p=0$, the right-hand side of the above sum rule is replaced by $i$ due to the $k=0$ pole of the GF [6]. For $p \neq 0$, however, the GF has no pole at $k=0$ corresponding to $\omega= \pm p c$ as demonstrated in Fig. 1. Using Eq. (14), one can write Eq. (10) as

$$
G_{k}\left(z, z^{\prime}\right)=\sum_{n} \frac{E_{n}(z) E_{n}\left(z^{\prime}\right)}{2 k_{n}}\left[\frac{1}{k-k_{n}}+F(k)\right],
$$

where $F(k)$ is an arbitrary function which will appropriately be chosen later in order to linearize a resulting matrix eigenvalue problem of the RSE.

We now consider an arbitrary perturbation $\Delta \varepsilon(z)$ of the dielectric constant inside the layer $|z|<a$. The new perturbed GF $\mathcal{G}_{k}\left(z, z^{\prime}\right)$ is related to the unperturbed one via the Dyson equation,

$$
\begin{aligned}
\mathcal{G}_{k}\left(z, z^{\prime}\right)= & G_{k}\left(z, z^{\prime}\right)-\left(k^{2}+p^{2}\right) \\
& \times \int_{-a}^{a} G_{k}\left(z, z^{\prime \prime}\right) \Delta \varepsilon\left(z^{\prime \prime}\right) \mathcal{G}_{k}\left(z^{\prime \prime}, z^{\prime}\right) d z^{\prime \prime} .
\end{aligned}
$$

Substituting Eq. (15) into Eq. (16) and using a similar spectral representation for the perturbed GF in terms of the perturbed modes $\mathcal{E}_{v}(z)$, in Eq. (16) we equate, following Ref. [6], residua at the perturbed poles $k=\varkappa_{v}$. This results in the following relationship between unperturbed and perturbed modes:

$$
\begin{aligned}
\mathcal{E}_{v}(z)= & -\left(\varkappa_{v}^{2}+p^{2}\right) \sum_{n} \frac{E_{n}(z)}{2 k_{n}}\left[\frac{1}{\varkappa_{v}-k_{n}}+F\left(\varkappa_{v}\right)\right] \\
& \times \int_{-a}^{a} E_{n}\left(z^{\prime}\right) \Delta \varepsilon\left(z^{\prime}\right) \mathcal{E}_{v}\left(z^{\prime}\right) d z^{\prime} .
\end{aligned}
$$

Note that the perturbed modes $\mathcal{E}_{v}(z)$ satisfy Eq. (4) with $\varepsilon(z)$ replaced by $\varepsilon(z)+\Delta \varepsilon(z)$ and the BCs Eq. (11) with $k_{n}$ replaced by $\varkappa_{v}$. In the interior region $|z|<a$ which contains the perturbation, the perturbed RSs can be expanded into the unperturbed ones, exploiting the completeness of the latter,

$$
\mathcal{E}_{v}(z)=\sum_{n} b_{n v} E_{n}(z)
$$

Substituting this expansion into Eq. (17) and equating coefficients at the same basis functions, $E_{n}(z)$, results in the matrix equation,

$$
b_{n v}=-\frac{\varkappa_{v}^{2}+p^{2}}{2 k_{n}}\left[\frac{1}{\varkappa_{v}-k_{n}}+F\left(\varkappa_{v}\right)\right] \sum_{m} V_{n m} b_{m v},
$$

where

$$
V_{n m}=\int_{-a}^{a} \Delta \varepsilon(z) E_{n}(z) E_{m}(z) d z
$$

is the matrix of the perturbation in the basis of unperturbed RSs.

Equation (19) is a matrix eigenvalue problem which can be solved numerically in order to find the wave vectors $\varkappa_{\nu}$ and the corresponding eigenfrequencies of the perturbed RSs as well as their expansion coefficients $b_{n v}$ in terms of the unperturbed ones. However, this problem is generally nonlinear in $\varkappa_{v}$ as can be seen by choosing $F(k)=0$. Nonlinear eigenvalue problems are known to lead to numerical instabilities and can produce spurious solutions. In order to avoid these issues, we choose

$$
F(k)=-\frac{k}{k^{2}+p^{2}}=-\frac{k c^{2}}{\omega^{2}},
$$

explicitly depending on the in-plane wave vector $p$, which linearizes the eigenvalue problem. Indeed, with the substitution $c_{n v}=b_{n v} \sqrt{k_{n} / \varkappa_{v}}$, the eigenvalue problem is given by

$$
\begin{aligned}
& \sum_{m}\left(\frac{\delta_{n m}}{k_{n}}+\frac{V_{n m}}{2 \sqrt{k_{n} k_{m}}}\right) c_{m v} \\
& =\frac{1}{\varkappa_{v}} \sum_{m}\left(\delta_{n m}-\frac{p^{2} V_{n m}}{2 k_{n} \sqrt{k_{n} k_{m}}}\right) c_{m v},
\end{aligned}
$$

which is linear and can be solved by inverting the matrix on the right-hand side of Eq. (22) and diagonalizing the resulting nonsymmetric matrix on the left-hand side in order to obtain 
its eigenvalues $1 / \varkappa_{\nu}$. Alternatively, one can solve Eq. (22) by employing a variety of software libraries available for generalized linear matrix eigenvalue problems. Note that the matrix equation of the RSE for normal incidence previously derived in Ref. [6] is restored by choosing $p=0$ in Eq. (22).

\section{RESULTS}

To apply the method developed in Sec. III, we first construct a convenient basis of unperturbed states. We use the RSs of a homogeneous dielectric slab discussed in Sec. II. We calculate the wave functions $E_{n}(z)$ of the RSs and investigate the dependence of their eigenvalues $k_{n}$ on the in-plane wave vector $p$. Then, using the RSE, in particular, Eqs. (20) and (22), we calculate the perturbed eigenvalues $\varkappa_{\nu}$ for the simplest perturbation, which is constant across the slab, and compare the RSE results with the available exact solution. Finally, we use the RSE to treat a structured perturbation simulating a Bragg-mirror MC. We specifically discuss the lowest-energy cavity mode (CM) and compare results with the transfer-matrix calculation of the MC transmission and with an available analytic approximation for the CM linewidth.

\section{A. Unperturbed resonant states}

The solutions of Eq. (4) which satisfy the outgoing-wave BCs Eq. (11) in TE polarization take the form

$$
E_{n}(z)= \begin{cases}(-1)^{n} A_{n} e^{-i k_{n} z}, & z<-a, \\ B_{n}\left[e^{i q_{n} z}+(-1)^{n} e^{-i q_{n} z}\right], & |z| \leqslant a, \\ A_{n} e^{i k_{n} z}, & z>a,\end{cases}
$$

where the eigenvalues $k_{n}$ satisfy the secular equation,

$$
\left(k_{n}-q_{n}\right) e^{i q_{n} a}+(-1)^{n}\left(k_{n}+q_{n}\right) e^{-i q_{n} a}=0,
$$

with $q_{n}=\sqrt{\epsilon_{s} k_{n}^{2}+\left(\epsilon_{s}-1\right) p^{2}}$. Here we use an integer index $n$ which takes even (odd) values for symmetric (antisymmetric) $E_{n}(z)$, respectively. The normalization constants $A_{n}$ and $B_{n}$ are found from the continuity of $E_{n}$ across the boundaries and the normalization condition Eq. (12). They take the form

$$
\begin{gathered}
A_{n}=\frac{e^{-i k_{n} a}}{\sqrt{a\left(\epsilon_{s}-1\right)}} \sqrt{\frac{\epsilon_{s} \omega_{n}^{2} / c^{2}-p^{2}}{\epsilon_{s} \omega_{n}^{2} / c^{2}+i p^{2} /\left(k_{n} a\right)}}, \\
B_{n}=\frac{(-i)^{n}}{2 \sqrt{a \epsilon_{s}+i p^{2} /\left(k_{n} \omega_{n}^{2} / c^{2}\right)}},
\end{gathered}
$$

where $\omega_{n}^{2} / c^{2}=k_{n}^{2}+p^{2}$.

The frequencies $\omega_{n}$ of the RSs of a dielectric slab for $p a=5$ and $\epsilon_{s}=9$ were shown in Fig. 1 . The normal wave vectors $k_{n}$ of the RSs for a slab with $\epsilon_{s}=3$ versus $p$ are given in Fig. 4. All states in the range $\left|\operatorname{Re} k_{n} a\right|<5$ and $\left|\operatorname{Im} k_{n} a\right|<5$ for $p a<5$ are shown in Fig. 4(a) and are separated into mode types in Figs. 4(b) and 4(c). For WG and AWG modes, Re $k_{n}=0$, therefore, Fig. 4(c) shows only their imaginary parts, which are positive for WG modes, corresponding to evanescent waves, and negative for AWG modes, corresponding to exponentially growing waves outside the slab. The WG and AWG modes continuously transform into each other and produce branches similar to those also seen for FP modes. These branches cross each other at certain points [shown in Figs. 4(b) and 4(c) by
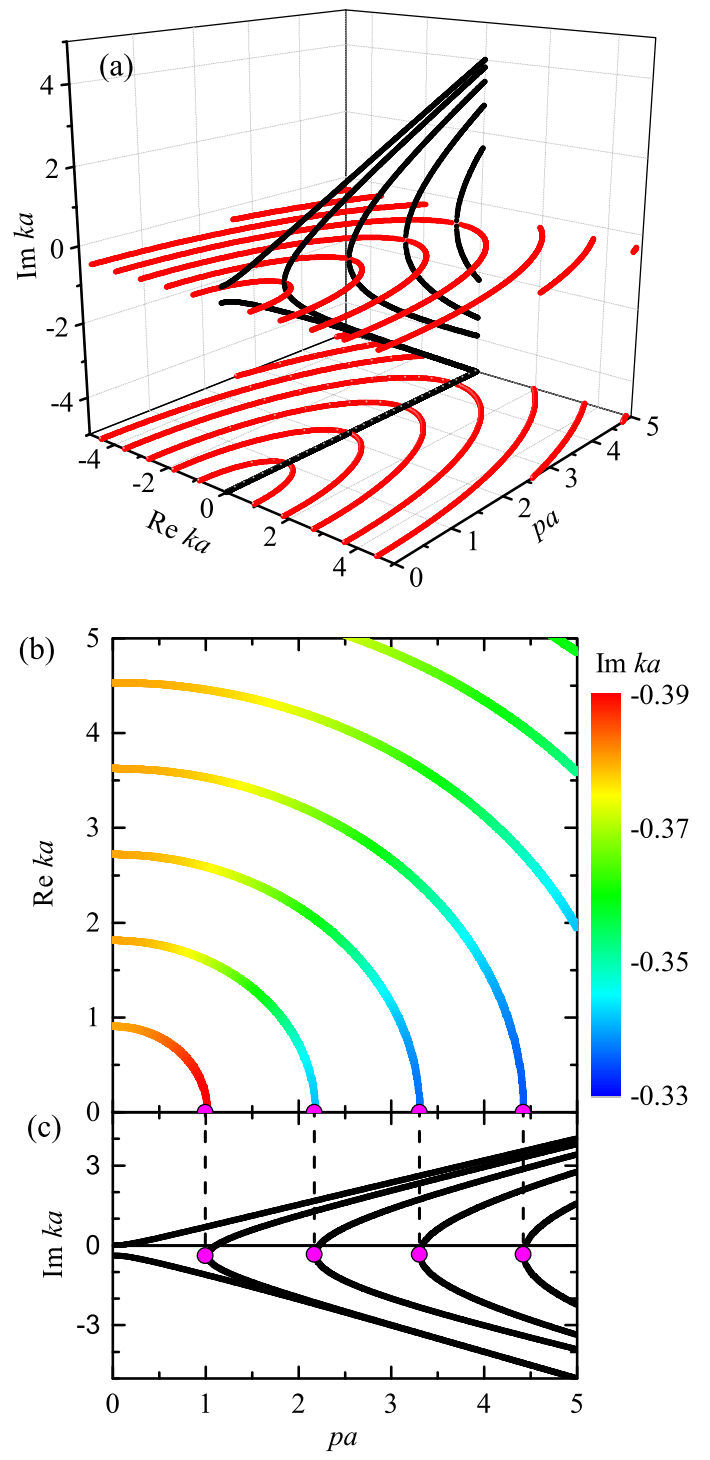

FIG. 4. (Color online) Resonant-state wave numbers of a homogenous dielectric slab with $\epsilon_{s}=3$ as a function of the in-plane wave vector $p$ : (a) The complex wave vectors $k_{n}$ of Fabry-Pérot (red lines) and WG and AWG modes (black lines) with a projection on the lower plane, (b) Re $k_{n}$ of Fabry-Pérot modes with the color giving the value of $\operatorname{Im} k_{n}$, and (c) $\operatorname{Im} k_{n}$ of the WG and AWG modes. The points where the modes in panels (b) and (c) connect are given by magenta dots joined by dashed lines.

magenta dots] where two FP modes are transformed into two AWG modes which subsequently transform into a AWG - WG mode pair. The AWG mode branch, which starts at $p=0$, has no connection to any WG or FP branches; the mode on this branch was identified in Fig. 1 as the leaky mode.

The RSs of the homogeneous slab, similar to those shown in Fig. 4, are used as a basis for the RSE in the two examples given below. In general, for any local perturbation $\Delta \varepsilon(z)$ which does not change the translational symmetry of the slab, i.e., does not depend on $x$ or $y$, the in-plane momentum $p$ remains a good quantum number. In other words, $\Delta \varepsilon(z)$ does not mix states with different $p$ 's so that, in any such problem solved 
by the RSE, one can use the basis of RSs with a given fixed value of $p$.

The numerical results shown in the following were obtained with a program written in C++ using the Numerical Algorithms Group library, running on an Intel E8500 CPU, taking a few seconds for a given $p$ with a basis size of $N=500$. The matrix elements of the perturbations were calculated using the analytic expressions provided in the Appendix of Ref. [7].

\section{B. Full-width perturbation}

To illustrate the accuracy and convergence of the RSE, we consider a homogeneous full-width perturbation of the slab, which is given by

$$
\Delta \varepsilon(z)= \begin{cases}\Delta \epsilon & \text { for }|z| \leqslant a, \\ 0 & \text { otherwise }\end{cases}
$$

and for which the exact solution can be obtained by solving the transcendental Eq. (24) with $\epsilon_{s}$ replaced by $\epsilon_{s}+\Delta \epsilon$. We

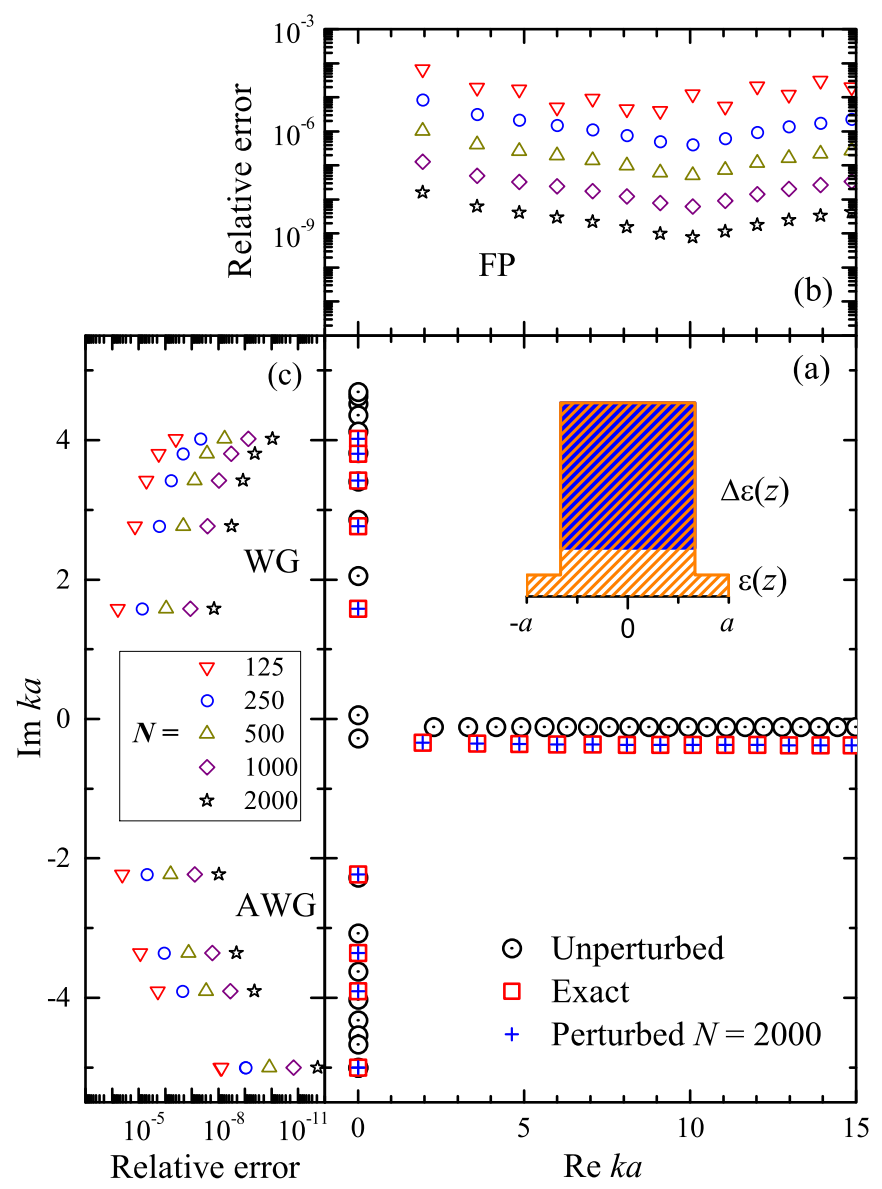

FIG. 5. (Color online) (a) Exact (squares) and calculated by the RSE with $N=2000$ (crosses) resonant-state wave numbers of a homogeneous dielectric slab with $\epsilon_{s}=3$ along with those of the unperturbed slab with $\epsilon_{s}=9$ (circles with a dot). Relative errors in the calculation of (b) Fabry-Pérot modes and (c) waveguide and antiwaveguide modes for different total numbers of basis states $N$ used in the RSE as labeled. Inset: the dielectric constant profile of the unperturbed and perturbed systems with the full-width homogeneous perturbation of the slab $\Delta \epsilon=-6$. denote these exact perturbed wave numbers as $\varkappa_{\nu}^{\text {(exact) }}$ and compare them with the perturbed values $\varkappa_{v}$ obtained by using the RSE for different basis sizes $N$. We choose, as the basis of given size, all poles with $\left|k_{n}\right|<k_{\max }(N)$ using a suitably chosen wave-number cutoff $k_{\max }(N)$.

In Fig. 5, we compare the RSE wave numbers with the exact wave numbers for our system in the case of $p a=5$ and $\Delta \epsilon=-6$. In Fig. 5(a), we can see that the RSE is reproducing the exact solution to a high accuracy, which is quantified by the relative error $\left|\varkappa_{v} / \varkappa_{v}^{\text {(exact) }}-1\right|$ shown in Fig. 5(b) for the FP modes with $\operatorname{Re} \varkappa_{v}>0$ and in Fig. 5(c) for the WG and AWG modes. We see that the relative error scales as $N^{-3}$, which was observed also in planar systems at normal incidence [6,7] and in homogeneous microcylinders [8] and microspheres [6]. In the simulation used to generate Fig. 5 for a basis of $N=2000$, we find that the RSE reproduces about 300 modes with a relative error below $10^{-8}$. This error can further be improved by 1 to 2 orders of magnitude using the extrapolation method described in Ref. [7].

\section{Microcavity perturbation}

To evaluate the RSE for inclined geometry in the presence of sharp resonances in the optical spectrum, we use a Braggmirror MC, which consists of a FP cavity of thickness $L_{C}$ and dielectric constant $\epsilon_{C}=9$ surrounded by distributed Bragg reflectors (DBRs). The DBRs consist of $P=5$ pairs of dielectric layers with alternating high $\epsilon_{H}=9$ and low $\epsilon_{L}=$ 2.25 susceptibilities as illustrated by the inset in Fig. 6 . The alternating layers have a quarter-wavelength optical thickness, and the cavity has a half-wavelength optical thickness. The nominal wavelength which determines the layer thickness is that of the lowest-frequency $\mathrm{CM}$ at normal incidence. As the unperturbed system, we used a dielectric slab with $\epsilon_{s}=9$ as in Sec. IV B.

The unperturbed modes of the slab and the perturbed modes of the MC are shown in Fig. 6(a) for $p a=5$. One can see how the nearly equidistant FP modes of the unperturbed system are redistributed in the $\mathrm{MC}$, transforming into a sharp $\mathrm{CM}$ in the middle of a wide stop band and modes outside of the stop band. The link between the peaks in the transmission in Fig. 6(b) and the poles in Fig. 6(a) is also exemplified by the real part of the poles giving the position of the peaks in transmission and the imaginary part giving their linewidth. This is discussed in Ref. [7] in terms of the GF, which is related to the transmission via $T(k)=2 i k G_{k}(a,-a) e^{-2 i k a}$.

The transmission $T(k)$ for a layered planar structure can be calculated using the transfer-matrix method leading to the following explicit result:

$$
T(k)=\frac{e^{-i\left(q_{0}+q_{M}\right) a}}{\xi_{M}^{+}}
$$

in which $\xi_{M}^{+}$is found from the recursive formula,

$$
2 \xi_{j+1}^{ \pm}=\left(1 \pm \frac{q_{j+1}}{q_{j}}\right) e^{-i q_{j} a_{j}} \xi_{j}^{+}+\left(1 \mp \frac{q_{j+1}}{q_{j}}\right) e^{i q_{j} a_{j}} \xi_{j}^{-},
$$




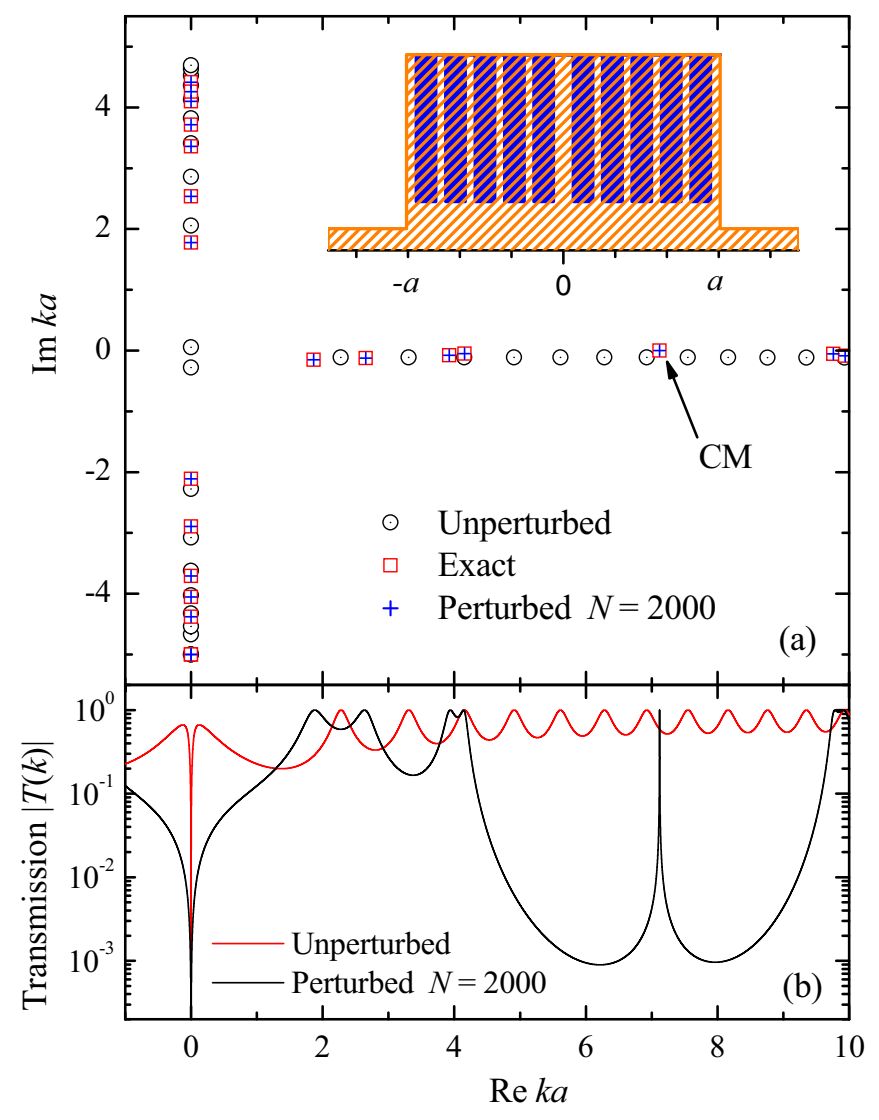

FIG. 6. (Color online) (a) The same as in Fig. 5(a) but with the perturbed system being the Bragg-mirror microcavity with the dielectric profile shown in the inset. The lowest-energy cavity mode is shown by an arrow. (b) Transmission as a function of the normal component of the wave vector $k$ for the perturbed (thick black curve) and unperturbed systems (thin red curve) demonstrating the correspondence between the RS wave numbers in panel (a) and the peaks in the transmission.

with the starting value,

$$
2 \xi_{1}^{ \pm}=\left(1 \pm \frac{q_{1}}{q_{0}}\right)
$$

and the normal component of the wave vector in the $j$ th layer,

$$
q_{j}=\sqrt{\epsilon_{j} k^{2}+\left(\epsilon_{j}-1\right) p^{2}} .
$$

Here $\epsilon_{j}$ and $a_{j}$, respectively, are the dielectric constant of the $j$ th layer and its width so that $\sum_{j=1}^{M-1} a_{j}=2 a$. The layers $j=0$ and $j=M$, respectively, correspond to the vacuum before and after the MC so that $q_{0}=q_{M}=k$ and $q_{j} \geq 0$ for real $\epsilon_{j} . M$ gives the total number of interfaces in the structure; in the present case, $M=2(2 P+1)$.

In Fig. 7, we show the evolution of the perturbed poles with $p$. We see that one of the modes is separated in the middle of a gap and has an imaginary part well below the others. This mode is know as the CM. The perturbed Green's function $\mathcal{G}_{k}\left(z, z^{\prime}\right)$, which has a spectral representation equivalent to Eq. (10), and the corresponding transmission $T(k)$ are dominated by the single term from the $\mathrm{CM}$ in this frequency region, therefore, a sharp isolated peak is seen in the center of the stop band in

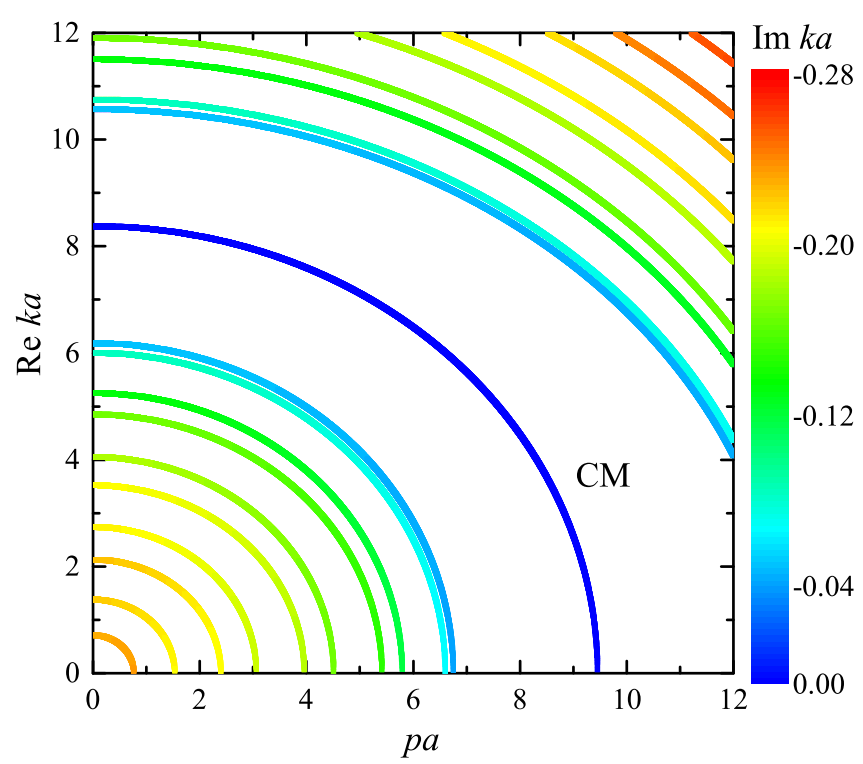

FIG. 7. (Color online) The same as in Fig. 4(b) but for the Braggmirror microcavity with the dielectric profile given by the inset in Fig. 6(a).

Fig. 6(b). Interestingly, the modes in Fig. 7 show an almost circular behavior, indicating that the frequency of each mode $\omega_{\nu}=c \sqrt{\varkappa_{v}^{2}+p^{2}}$ is approximately constant versus angle $\theta$.

Indeed, in Fig. 8(a), we can see that the CM frequency $\omega_{C}$ has a weak dependence on $\theta$, whereas, the corresponding wave vector $\varkappa_{C}$ changes more strongly. In parallel, the linewidth given in Fig. 8(b) shows a similar behavior both in the $\omega$ and in the $k$ representations, although, at $\theta \rightarrow \pi / 2$, the imaginary part of $\omega_{C}$ is 1 order of magnitude smaller than that of $\varkappa_{C}$.

Figures 8(a) and 8(b) demonstrate good agreement between $\varkappa_{C}$ obtained using the RSE and $\varkappa_{C}^{\text {(exact) }}$ extracted from the linewidth in the transmission calculated via Eqs. (28)-(31). Figure $8(\mathrm{c})$ shows the relative error $\left|\varkappa_{C} / \varkappa_{C}^{\text {(exact) }}-1\right|$ for different values of $N$ demonstrating convergence of the RSE for the cavity mode with $N^{-3}$, the same as for the homogeneous perturbation of the slab. The convergence behavior depends on the distribution of the perturbation in the wave-vector space as discussed in Refs. [7,8]. Interestingly, the RSE can reproduce sharp resonances in the transmission profile, despite the absence of sharp resonances in the basis.

We can also compare the results in Fig. 8(b) with an analytic approximation for the CM linewidth,

$$
\operatorname{Im} \omega_{C}=-\frac{2 c \eta_{\mathrm{ext}}}{n_{C} \eta_{C}} \frac{\left(\eta_{L} / \eta_{H}\right)^{2 P}}{L_{C} \cos \left(\theta_{C}\right)+\frac{\lambda_{C}}{2} \frac{\eta_{L} \eta_{H}}{\eta_{H}-\eta_{L}} \frac{1}{\eta_{C}}},
$$

which we have derived by generalizing the approximation for normal incidence of light available in the literature $[7,31,32]$. Here $n_{j}$ is the refractive index of layer $j, \eta_{j}=n_{j} \cos \left(\theta_{j}\right)$, and $\theta_{j}$ is the angle to the normal in layer $j$, given by $n_{j} \sin \left(\theta_{j}\right)=n_{\text {ext }} \sin (\theta)$. The layers $j$ used are as follows: the external region (ext), which is vacuum in our case, the high-index $(H)$ layer, the low-index $(L)$ layer of the Bragg mirror, and the cavity layer $(C)$. The cavity wavelength is given by $\lambda_{C}=2 L_{C} \cos \left(\theta_{C}\right)$. Equation (32) is exact in the limit $P \rightarrow \infty$ for a structure with Bragg-mirror layer widths strictly 


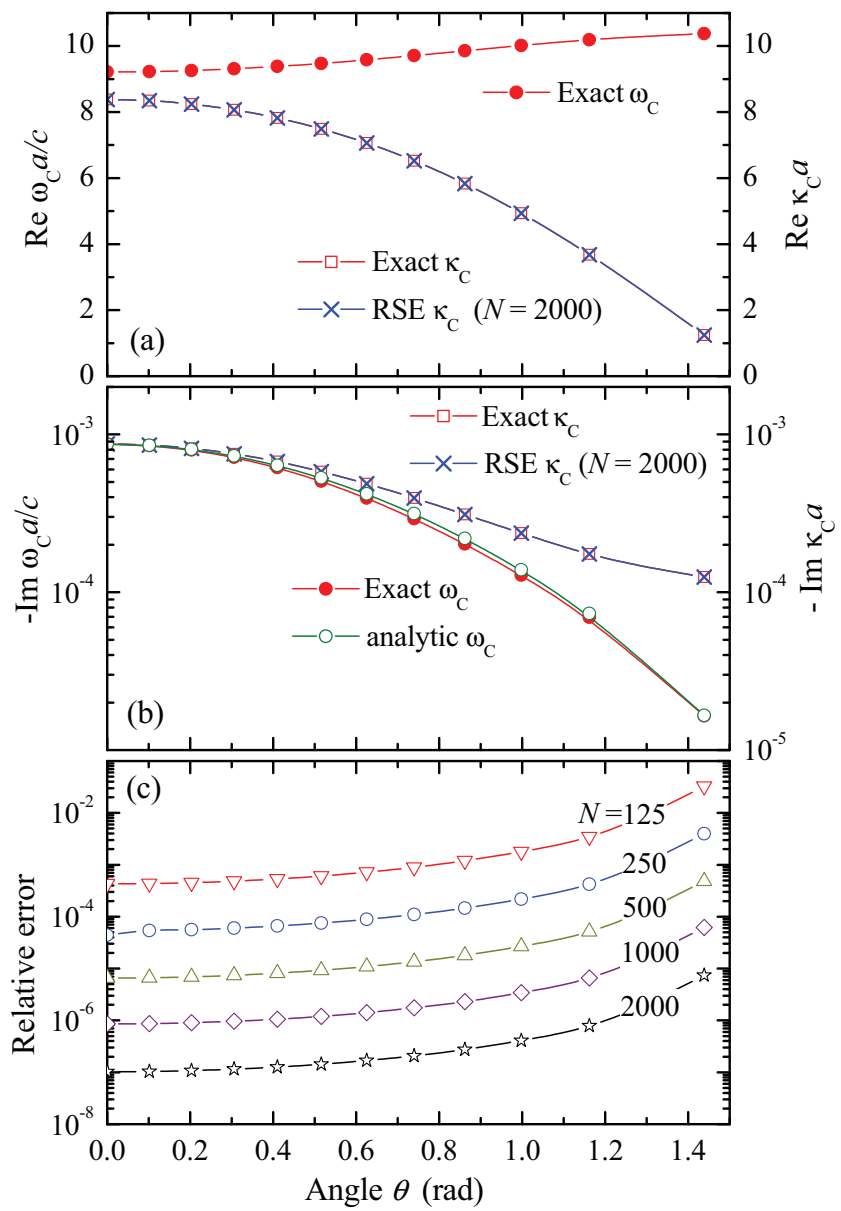

FIG. 8. (Color online) (a) Real and (b) imaginary parts of the cavity mode frequency $\omega_{C}$ (left axes) and the normal component of the wave vector $\varkappa_{C}$ (right axes) calculated using the RSE (blue crosses) for $N=2000$, the transfer-matrix method (red circles and open squares), and the analytic approximation Eq. (32) for the linewidth $\Gamma=-\operatorname{Im} \omega_{C}$ (green open circles). (c) Relative error of $\varkappa_{C}$ determined by the RSE for different basis sizes $N$ as given. All data are shown as a function of the angle of incidence $\theta$, and all symbols are connected by lines as a guide to the eye. equal to a quarter-wavelength and the cavity layer width equal to a half-wavelength optical thickness. This condition depends on the incident angle and, in our fixed structure, is fulfilled for normal incidence only. Nevertheless, Eq. (32) reproduces the exact result reasonably well over the whole angle range as shown in Fig. 8(b).

\section{CONCLUSION}

We have generalized the resonant-state expansion to planar optical systems with inclined geometry. The method is based on the spectral representation of the Green's function of Maxwell's wave equation and expansion of the optical modes of a perturbed system into a complete set of resonant states of a simple dielectric slab. In inclined geometry, the spectrum of a planar system contains a continuum of resonances originating from a cut of the Green's function, which we have eliminated by mapping the frequency into the normal wave vector. The optical modes and spectra of a perturbed planar system are then calculated by solving a linear matrix eigenvalue problem containing matrix elements of the perturbation in the basis of discrete resonant states only. We have verified the method on full-width homogeneous and Bragg-mirror microcavity perturbations and have compared results with obtained analytic solutions, demonstrating fast convergence of the method towards the exact result.

We expect that the method of eliminating the cut presented here will be suited to treat planar photonic crystals and cavities. We have recently demonstrated application of the RSE to twodimensional open optical systems at normal incidence. The present method will also enable extending this treatment to inclined geometry and will provide an efficient algorithm for calculating the optical modes in fibers and waveguides, such as photonic crystal fibers with complex structures.

\section{ACKNOWLEDGMENTS}

M.B.D. acknowledges support by the EPSRC under the DTA scheme. E.A.M. acknowledges partial support from RFBR Grant No. 12-02-00795.
[1] J. C. Knight, Nature (London) 424, 847 (2003).

[2] S. John, Nature (London) 460, 337 (2009).

[3] J. C. Cervantes-Gonzalez, D. Ahn, X. Zheng, S. K. Banerjee, A. T. Jacome, J. C. Campbell, and I. E. Zaldivar-Huerta, Appl. Phys. Lett. 101, 261109 (2012).

[4] A. J. Siegert, Phys. Rev. 56, 750 (1939).

[5] L. A. Weinstein, Open Resonators and Open Waveguides (Golem, Boulder, CO, 1969).

[6] E. A. Muljarov, W. Langbein, and R. Zimmermann, Europhys. Lett. 92, 50010 (2010).

[7] M. B. Doost, W. Langbein, and E. A. Muljarov, Phys. Rev. A 85, 023835 (2012).

[8] M. B. Doost, W. Langbein, and E. A. Muljarov, Phys. Rev. A 87, 043827 (2013).

[9] M. B. Doost, W. Langbein, and E. A. Muljarov, arXiv:1403.1609.
[10] L. Chantada, N. I. Nikolaev, A. L. Ivanov, P. Borri, and W. Langbein, J. Opt. Soc. Am. B 25, 1312 (2008).

[11] R. Dubertrand, E. Bogomolny, N. Djellali, M. Lebental, and C. Schmit, Phys. Rev. A 77, 013804 (2008).

[12] C. P. Dettmann, G. V. Morozov, M. Sieber, and H. Waalkens, Europhys. Lett. 87, 34003 (2009).

[13] L. Collot, V. Lefevre-Seguin, B. Brune, J. M. Raimond, and S. Haroche, Europhys. Lett. 23, 327 (1993).

[14] A. Taflove and S. C. Hagness, Computational Electrodynamics: The Finite-Difference Time-Domain Method, 2nd ed. (Artech House, Norwood, MA, 2000).

[15] S. C. Hagness, D. Rafizadeh, S. T. Ho, and A. Taflove, J. Lightwave Technol. 15, 2154 (1997).

[16] J. Wiersig, J. Opt. 5, 53 (2003).

[17] O. C. Zienkiewicz and R. L. Taylor, The Finite Element Method, 5th ed. (Butterworth-Heinemann, Oxford, 2000). 
[18] B. M. A. Rahman, F. A. Fernandez, and J. B. Davies, Proc. IEEE 79, 1442 (1991).

[19] B. N. Jang, J. Wu, and L. Povinelli, J. Comput. Phys. 125, 104 (1996).

[20] A. V. Boriskin, S. V. Boriskina, A. Rolland, R. Sauleau, and A. I. Nosich, J. Opt. Soc. Am. A 25, 1169 (2008).

[21] L. C. Andreani and D. Gerace, Phys. Rev. B 73, 235114 (2006).

[22] V. Savona, Phys. Rev. B 83, 085301 (2011).

[23] D. M. Whittaker and I. S. Culshaw, Phys. Rev. B 60, 2610 (1999).

[24] S. G. Tikhodeev, A. L. Yablonskii, E. A. Muljarov, N. A. Gippius, and T. Ishihara, Phys. Rev. B 66, 045102 (2002).
[25] R. Newton, J. Math. Phys. 1, 319 (1960).

[26] R. M. More, Phys. Rev. A 4, 1782 (1971).

[27] J. B. Conway, Functions of One Complex Variable I, 2nd ed. (Springer-Verlag, New York, 1978).

[28] R. Zavin and N. Moiseyev, J. Phys. A 37, 4619 (2004).

[29] For the TM polarization, the results are similar, however, one has to take into account the vectorial form of the electric field in this case as done, e.g., in Ref. [9].

[30] A strict derivation of Eqs. (10) and (12) for an arbitrary $\varepsilon(z)$ is similar to that given for $p=0$ in the Appendix of Ref. [6].

[31] L. C. Andreani, Phys. Lett. A 192, 99 (1994).

[32] V. Savona, L. C. Andreani, P. Schwendimann, and A. Quattropani, Solid State Commun. 93, 733 (1995). 